\section{Transthoracic ultrasound shear wave elastography for the study of subpleural lung lesions}

\author{
Carla Maria Irene Quarato ${ }^{1}$, Mariapia Venuti ${ }^{1}$, Lucia Dimitri ${ }^{2}$, Donato Lacedonia', \\ Anna Simeone ${ }^{3}$, Antonio Mirijello ${ }^{4}$, Salvatore De Cosmo ${ }^{4}$, Evaristo Maiello ${ }^{5}$, \\ Marco Taurchini ${ }^{6}$, Giulia Scioscia ${ }^{1}$, Maria Pia Foschino Barbaro ${ }^{1}$, Massimiliano Copetti ${ }^{7}$, \\ Marco Sperandeo ${ }^{8}$ \\ ${ }^{1}$ Department of Medical and Surgical Sciences, Institute of Respiratory Diseases, Policlinico \\ Riuniti di Foggia, University of Foggia, Foggia; ${ }^{2}$ Unit of Pathology, ${ }^{3}$ Department of Radiology, \\ ${ }^{4}$ Department of Internal Medicine, ${ }^{5}$ Unit of Oncology, ${ }^{6}$ Unit of Thoracic Surgery, ${ }^{7}$ Unit of \\ Biostatistics, ${ }^{8}$ Unit of Interventional and Diagnostic Ultrasound, Department of Internal Medicine, \\ IRCCS Fondazione Casa Sollievo della Sofferenza, San Giovanni Rotondo, Foggia, Italy
}

Purpose: The aim of this study was to assess whether new-generation shear wave elastography (SWE) is suitable for the characterization of lung subpleural lesions.

Methods: In total, 190 consecutive patients with subpleural lung lesions received ultrasonography and SWE. Patients with suspected malignancy underwent ultrasound-guided transthoracic needle biopsy. Final diagnoses were made on the basis of patients' clinical course, microbiological studies, and histological results. SWE was also performed in 25 healthy volunteers.

Results: We found no statistically significant differences in stiffness between lung carcinomas, lung metastases, and pneumonia $(P=0.296)$ or between different histological types of lung cancer $(P=0.393)$. Necrosis was associated with reduced stiffness in pneumonia. Excluding necrotic lesions, pneumonia showed higher stiffness than lung carcinomas $(2.95 \pm 0.68 \mathrm{~m} / \mathrm{s}$ vs. $2.60 \pm 0.54 \mathrm{~m} / \mathrm{s}, P=0.006)$. Chronic pneumonia showed increased stiffness $(3.03 \pm 0.63 \mathrm{~m} /$ s), probably due to the presence of fibrotic tissue on histology. Pleural effusion was associated with a statistically significant reduction in stiffness, both in lung carcinomas $(P=0.004)$ and lung metastases $(P=0.002)$. The presence of air in healthy lung tissue may lead to incorrect speed estimates due to shear wave reflection (very high values, $14.64 \pm 2.19 \mathrm{~m} / \mathrm{s}$ ).

Conclusion: Transthoracic SWE could not distinguish lung malignancy from pneumonia, or between different histological types of lung carcinomas. In particular, SWE seems unable to resolve the clinical dilemma of chronic subpleural consolidations.

Keywords: Transthoracic ultrasound; Ultrasound elastography; Shear wave elastography;

Ultrasound-guided transthoracic needle biopsy; Subpleural lesions; Artifacts

Key points: Transthoracic ultrasound (TUS)-shear wave elastography (SWE) is not able to distinguish pulmonary malignancy from pneumonia. Different histological types of lung carcinomas cannot be differentiated based on TUS- SWE results. SWE in lungs may be influenced by several conditions including pleural effusion, fibrosis, necrosis, and air.

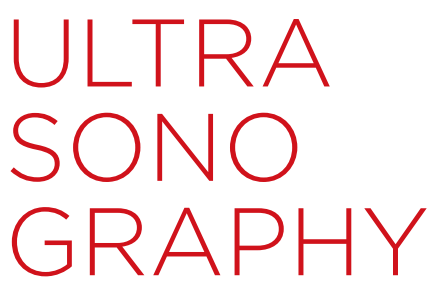

ORIGINAL ARTICLE

https://doi.org/10.14366/usg.21021 pISSN: 2288-5919 • elSSN: 2288-5943 Ultrasonography 2022;41:93-105

Received: January 29, 2021

Revised: March 22, 2021

Accepted: April 15, 2021

Correspondence to:

Carla Maria Irene Quarato, MD, Department of Medical and Surgical Sciences, Institute of Respiratory Diseases, Azienda OspedalieroUniversitaria Ospedale Riuniti di Foggia, University of Foggia, Foggia, Italy

Tel. +39-0881733038

Fax. +39-0881733038

E-mail: carlamariairene.quarato@ gmail.com

*This study was presented at the 2020 ERS International Congress, October 2020.

This is an Open Access article distributed under the terms of the Creative Commons Attribution NonCommercial License (http://creativecommons.org/ licenses/by-nc/4.0/) which permits unrestricted noncommercial use, distribution, and reproduction in any medium, provided the original work is properly cited.

Copyright @ 2022 Korean Society of Ultrasound in Medicine (KSUM)

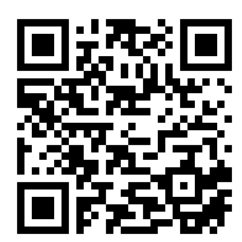

How to cite this article:

Quarato CMI, Venuti M, Dimitri L, Lacedonia D, Simeone A, Mirijello A, et al. Transthoracic ultrasound shear wave elastography for the study of subpleural lung lesions. Ultrasonography. 2022 Jan;41(1):93-105. 


\section{Introduction}

Transthoracic ultrasonography (TUS) can detect lung consolidations that are adherent to the pleural surface [1], and ultrasound-guided transthoracic needle biopsy (US-TNB) is a well-established and safe technique for the histological assessment of peripheral pulmonary nodules $[2,3]$. However, no specific ultrasound (US) pattern can distinguish inflammatory densities from solid cancer masses [1]. Therefore, persistent consolidations that fail to resolve by $50 \%$ in 2 weeks or completely in 4 weeks despite adequate antibiotic treatment [4] continue to pose a clinical dilemma. A persistent consolidation may be due to an inadequately treated or atypical infection, but may also hide a malignancy [5].

Ultrasound elastography (UE) is a novel technique that assesses tissue stiffness. This property can be described by Young's modulus, defined as: $E=\sigma / \varepsilon$, where $\sigma$ is the applied stress and $\varepsilon$ is the resultant deformation of the tissue. Therefore, UE may provide additional information on tissue mechanical properties compared to ultrasonography, which analyzes tissue acoustic properties. As the tissue stiffness of malignant lesions is usually higher than that of benign lesions, elastography could be used to differentiate malignancy from normal tissue, thus informing the decision of which nodules to sample during US-TNB.

There are two main methods for UE measurements. The first commercially available US scanners that incorporated tissue stiffness evaluation relied on the strain elastography (SE) method. SE estimates the qualitative relative strain of a target lesion in a region of interest (ROI) compared to that of the surrounding normal tissue via the stress generated by external compressions applied by the transducer. A pseudo-quantitative measurement, called the "strain ratio," is used, which is the ratio of strain measured in the reference normal tissue to the strain measured in the ROI: a strain ratio $>1$ indicates that the target lesion compresses less than the normal reference tissue (i.e., indicating greater stiffness) and vice versa.

In 2015 our group reported promising findings regarding the potential utility of SE for characterizing US-accessible lung nodules before reaching a histological diagnosis on US-TNB [6]. Cancer nodules displayed significantly lower elasticity than pneumonia consolidations, and the stiffness of squamous cell lung carcinoma was significantly higher than that of other primary lung cancers. However, a clear diagnostic cut-off for differentiating various histological types of lung cancer was not found.

Several technical confounders are known to affect the diagnostic accuracy of SE in the lung. A number of these can be traced back to general limitations of ultrasonography due to the air content of the normal pulmonary parenchyma; others are related to the operatordependent nature of the technique.
The new generation of sonoelastography scanners, equipped with shear wave elastography (SWE), do not require external compression; instead, they use a localized radiation force to induce a "push" inside the tissue of interest. This generates a shear wave that, propagating transversally, produces a shear wave front, which sweeps the image plane on both sides from the initial focal point of application. SWE equipment captures and calculates in real time the propagation speed of this transverse pressure wave that correlates with the elasticity of the tissues, traveling at greater speed in a rigid tissue than in a non-rigid one. The quantitative value of stiffness is obtained converting shear wave speed, measured in meters per second $(\mathrm{m} / \mathrm{s})$, to the Young modulus, which is expressed in kilopascals $(\mathrm{kPa})$, using the relationship $\mathrm{E}=3 \rho \mathrm{c}_{\mathrm{s}}$ in which $\rho$ represents tissue density, and $c_{s}$ represents speed [7].

SWE has been extensively applied to characterize liver fibrosis $[7,8]$, breast masses [9,10], prostate cancer lesions [11], thyroid nodules [12,13], cervical lymph nodes [14], and musculoskeletal soft tissues $[15,16]$. In these contexts, SWE displayed low variability with respect to SE [17]. With this background, the aim of the present study was to assess whether TUS-SWE may represent a new quantitative method that is also suitable for the study of lung subpleural lesions.

\section{Materials and Methods}

\section{Compliance with Ethical Standards}

This study was conducted in accordance with the amended Declaration of Helsinki. The local Institutional Ethics Committee of IRCCS Fondazione Casa Sollievo della Sofferenza, San Giovanni Rotondo (Italy) approved the protocol (TACE-CCS, n 106/2018) and participants provided informed written consent for all procedures, including biopsy.

\section{Study Design}

In this prospective comparative study, we examined by TUS and subsequent SWE analysis a cohort of patients presenting radiographic evidence of peripheral pulmonary lesions.

Patients presenting with shortness of breath or with difficulty in holding their breath and patients with peripheral pulmonary lesions not clearly displayed on ultrasonography were excluded from the study. As SWE examinations are contraindicated in pregnant women and persons with a history of pacemaker implantation [18], patients known to fall into these clinical categories were excluded. We also excluded patients with the following relative contraindications for US-TNB: an abnormal platelet number (i.e., platelet count $<30,000$ / $\mathrm{mm}^{3}$ ) or prolonged prothrombin time (i.e., a prothrombin timeinternational normalized ratio >1.5) and subpleural emphysema. 
From November 2018 to December 2019, 202 patients were assessed for eligibility. Five patients were excluded for contraindications (two patients had subpleural emphysema, two patients were unable to hold their breath, and one patient had abnormal blood clotting), two patients refused to participate, and five patients were excluded as they presented peripheral pulmonary lesions that were not evaluable on TUS. Finally, we enrolled 190 patients (118 men, 72 women; aged 65.88 \pm 6.33 years). In addition, SWE examinations were performed in 25 healthy volunteers. The inclusion criteria for healthy volunteers were the following: mean age comparable to that of the study group (i.e., 50-75 years), equal sex distribution (13 men, 12 women), no smoking habit, and no known active heart diseases or pleuro-pulmonary pathologies, in order to avoid the influence of pathologic and smoking-induced lung changes. A flow chart describing the patients included in the study is provided in Fig. 1.

Patients with known neoplasms or suspected to have malignant lesions were also pre-examined through a contrast-enhanced computed tomography (CT) scan according to the current diagnostic and staging protocol for lung cancer $[19,20]$. In patients with a clinical-radiological suspicion of infectious pneumonia, a contrastenhanced chest CT scan was performed after enrollment to further delineate the lesion(s). Lesions were categorized on the basis of the following contrast-enhanced CT findings: size, presence/absence of necrosis, presence/absence of air bronchogram, and presence/ absence of pleural effusion. In patients with multiple pulmonary lesions on a chest CT scan, the one that was most clearly seen on TUS was selected as the main target for subsequent examinations.

\section{TUS and TUS-SWE}

The TUS examination was performed with the patient in a sitting position using an Esaote MyLab-9 (Esaote-Biomedica, Genoa, Italy), equipped with QElaXto function. We used a convex multifrequency probe at 2-8 MHz, as it provides an adequate depth of penetration into the chest wall, a thoracic preset with depth varying between 70 and $140 \mathrm{~mm}$ and a tissue harmonic in order to reduce physiologically present artifacts. The time gain compensation was no more than $50 \%$. Conventional B-mode ultrasonography was used to localize the pulmonary lesion and categorize its morphology as follows: size, regular/irregular margins, homogeneous/inhomogeneous pattern, and presence/absence of pleural effusion.

Once the lung nodule had been sufficiently evaluated on conventional TUS, the image was frizzed and the QElaXto modality was activated. The box was positioned on a small, fixed ROI identified over the lesion on the two-dimensional B-mode grayscale image, and the radiation force was generated by pressing "ACQUIRE." Following the pulse, the subsequent perturbation of the tissue induced a shear wave moving laterally at a speed depending on the visco-elastic properties of the tissue. Ten consecutive shear wave measurements (in meters per second) were obtained in the $\mathrm{ROI}$, and were finally displayed on the monitor together with their median value and the interquartile range/median ratio (IQR/M).

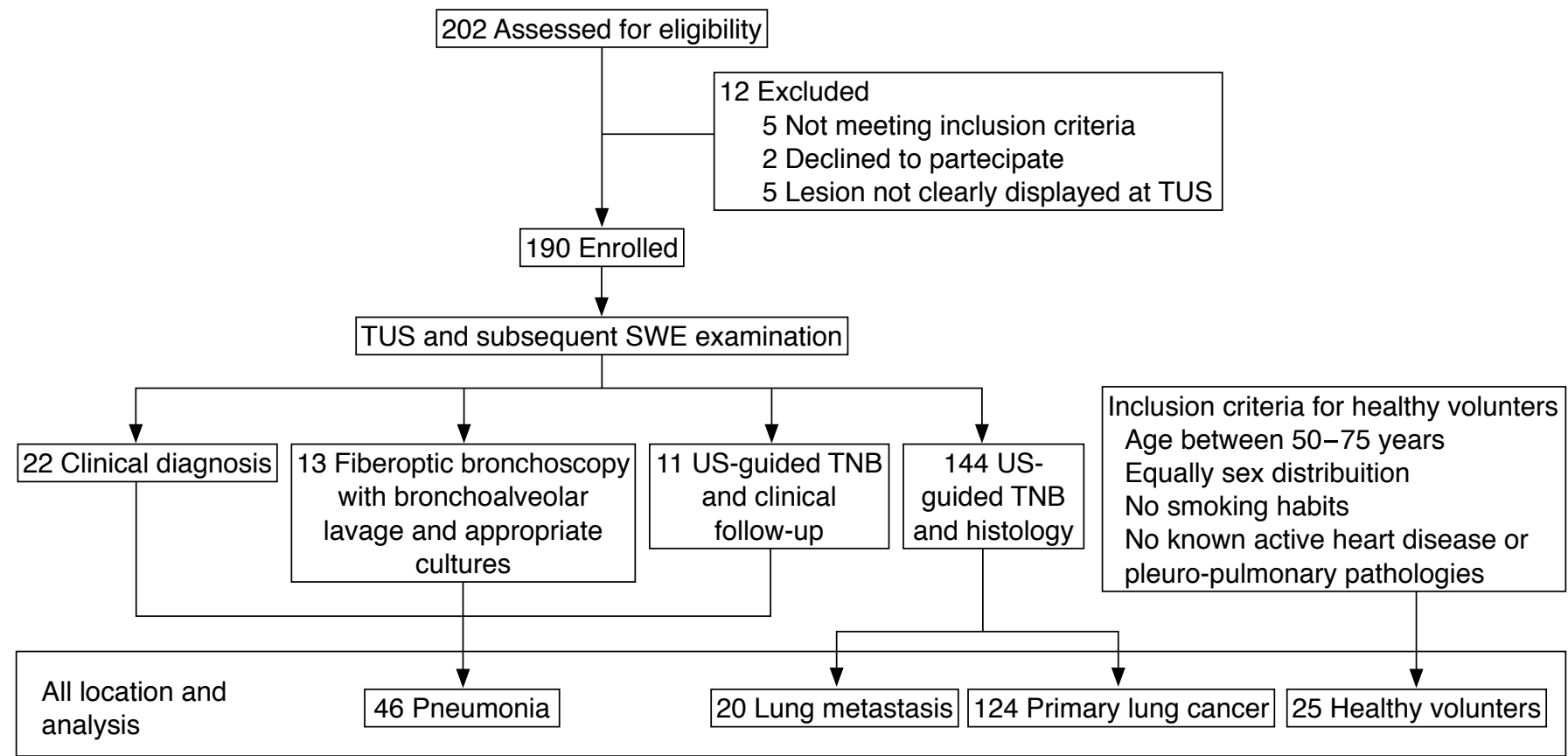

Fig. 1. Flow chart describing the patients included in the study. TUS, transthoracic ultrasonography; SWE, shear wave elastography; US, ultrasonography; TNB, transthoracic needle biopsy. 
The ROI stiffness measurement was defined as the median value of 10 measurements and was considered reliable if the IQR/M was less than $30 \%$. An automatic reliability tool discarded unreliable measurements, according to the statistical analysis of acquired signals. A three-dimensional shear wave quality map (eWave) provided an immediate feedback about tissue mechanical properties in the ROI: low-displacement regions (hard tissue) were displayed as blue and high-displacement regions (soft tissue) as red, while yellow, green, and light blue areas encoded intermediate degrees of stiffness. The SWE study aimed at the assessment of the whole lesion. For this purpose, at least 10 point ROls were randomly placed on the lesion area, at an average distance of $3 \mathrm{~cm}$ from the skin, with a depth of less than $4-5 \mathrm{~cm}$, as this alignment allows obtaining results with the lowest variability using a convex probe [21]. The mean value of these 10 point ROls was then calculated to obtain the final shear wave measurement (in meters per second) of the whole lesion. During each acquisition, the patient was instructed to hold his or her breath and the probe was positioned in the widest optimal area between the ribs and kept as perpendicular as possible to the skin.

Given the absence of target lesions in conventional US B-mode imaging, for the study of our 25 healthy volunteers the ROI was positioned at the chest wall/lung interface and SWE values were calculated as the average between the two medial-anterior and medial-posterior hemiclavicular and hemiscapular scans.

Conventional TUS and SWE examinations were performed and interpreted by three operators, who were blinded to the chest CT characteristics of each lesion and their diagnostic interpretation during the examinations. Inter-operator variability was assessed by another operator taking repeated SWE measures in the same subject 1 day apart. The coefficients of variation between the measurements were low $(0.6 \%-9.8 \%)$.

\section{US-TNB}

US-TNB was performed in 135 patients with lesions suspected of malignancy. The biopsies were performed by two expert operators (an internist and a thoracic surgeon) with over 30 years of experience in interventional ultrasound employing a multifrequency convex transducer (3.5-8 MHz) with a central opening for needle insertion and the modified Menghini technique. This technique consists of using an atraumatic 18-gauge needle with a Menghini type tip and a pyramidal stylet connected to a syringe plunger. By the help of a spring mechanism, the syringe plunger can be charged and automatically released.

The patient was instructed to hold his or her breath and the needle, with the syringe plunger charged, was advanced under realtime guidance in the lung. Once the lesion was reached, the syringe plunger was released, thus removing the stylet and applying suction. The needle was then pushed in and out of the lesion, thereby facilitating the ascent of pathological tissue. Biopsy specimens and cell blocks for both histology and cytology assessment were obtained (Fig. 2).

At the end of the biopsy procedure, the presence of the "sliding sign," which reflects movement of the pleura during respiratory excursion, was verified by real-time ultrasonography to rule out a pneumothorax [1]. Expiratory chest $X$-rays were also performed. Patients were closely monitored for 3-4 hours after the procedure.

\section{Final Diagnosis}

Final diagnoses were made on the basis of patients' clinical course, microbiological studies, and histological results. The microbiological studies included direct detection of microorganisms and appropriate cultures on bronchoalveolar lavage. In cases where bronchoscopy with microbiological evaluation was non-diagnostic, additional tissue sampling was performed. Histological diagnoses were made by a pathologist with 20 years of experience in lung diseases, blinded to the TUS and SWE findings. Lung lesions were grouped into three main categories: (1) primary lung carcinomas, (2) lung metastases, and (3) organizing pneumonia. Primary lung carcinomas were subtyped into (1) small cell lung cancer, (2) squamous cell carcinoma, (3) adenocarcinoma, and (4) large cell undifferentiated carcinoma.

\section{Statistical Analysis}

Descriptive statistics were produced for the demographic and imaging characteristics of the sample of patients enrolled in the study. Data were presented as means and standard deviations for continuous variables, and as number and percentage for categorical variables. The enrolled patients were divided into three groups according to their final diagnosis (lung carcinoma, lung metastases, and pneumonia) and compared in terms of demographic characteristics, TUS and chest CT findings, and SWE results. A subgroup analysis of SWE results was performed for different histological types of lung carcinomas and between usual community-acquired pneumonia (CAP) and CAP refractory to standard treatment (R-CAP). Another sub-analysis in terms of SWE results was performed between lesions with and without necrosis and lesions with and without associated pleural effusion. Analysis of variance and the unpaired Student $t$ test were used to assess differences between averages. For comparisons between more than two groups, the pairwise post-hoc Tukey test was performed to determine whether there was a difference between the means of all possible pairs. A $\mathrm{P}<0.05$ was considered to indicate statistical significance. 


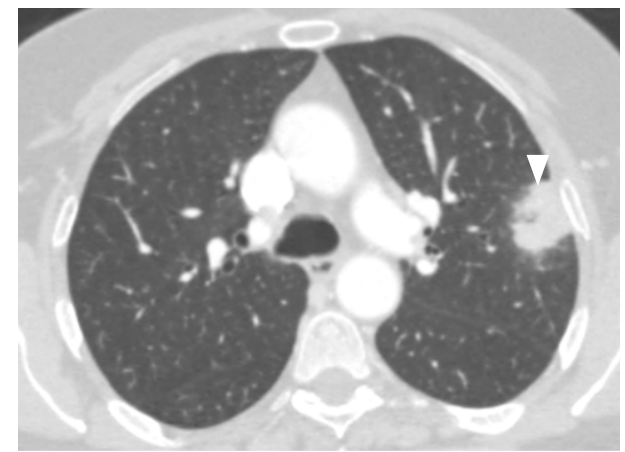

A

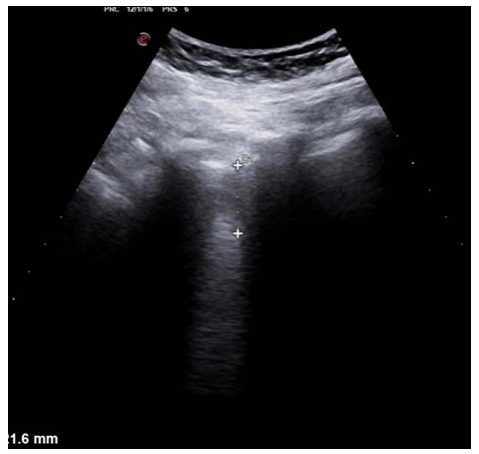

B



C

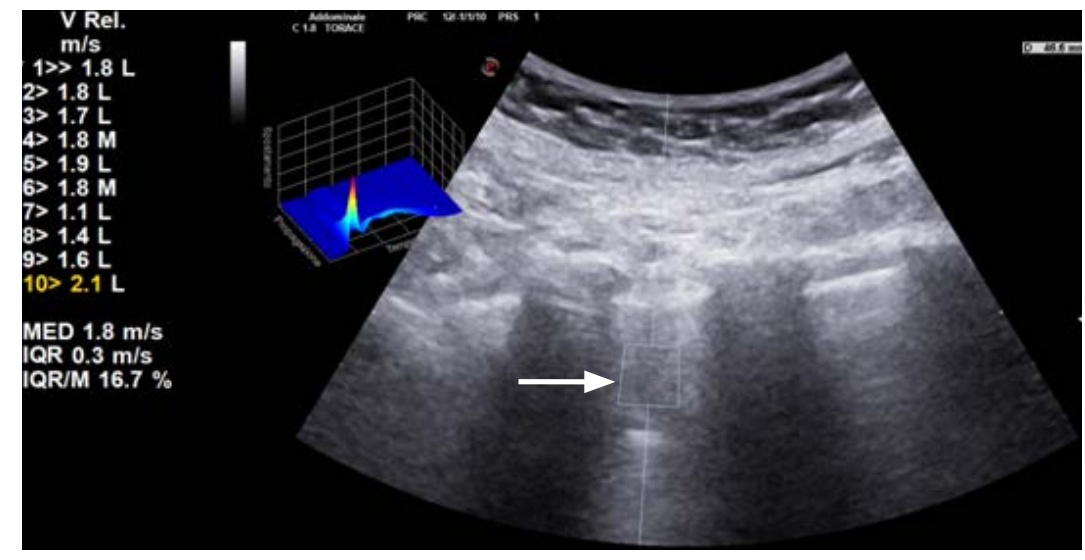

D

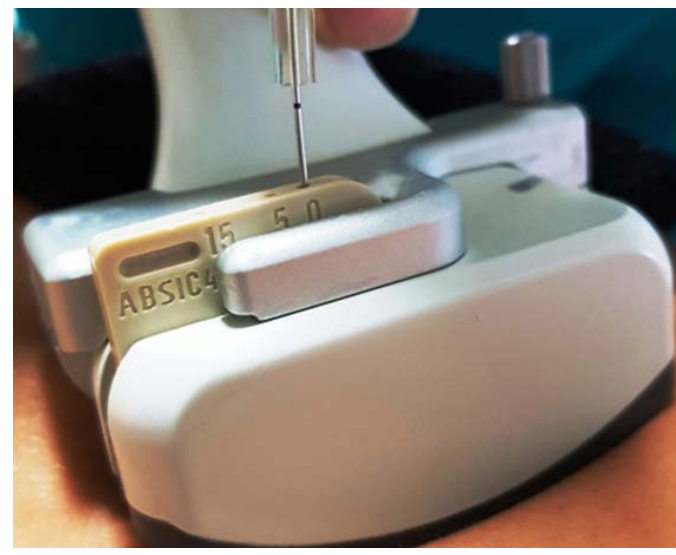

$E$

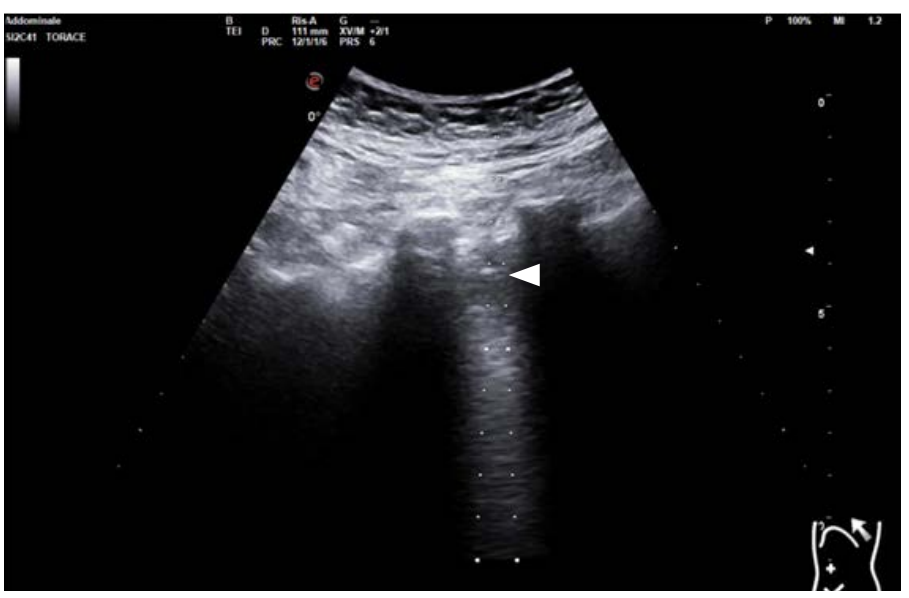

$$
\mathrm{F}
$$

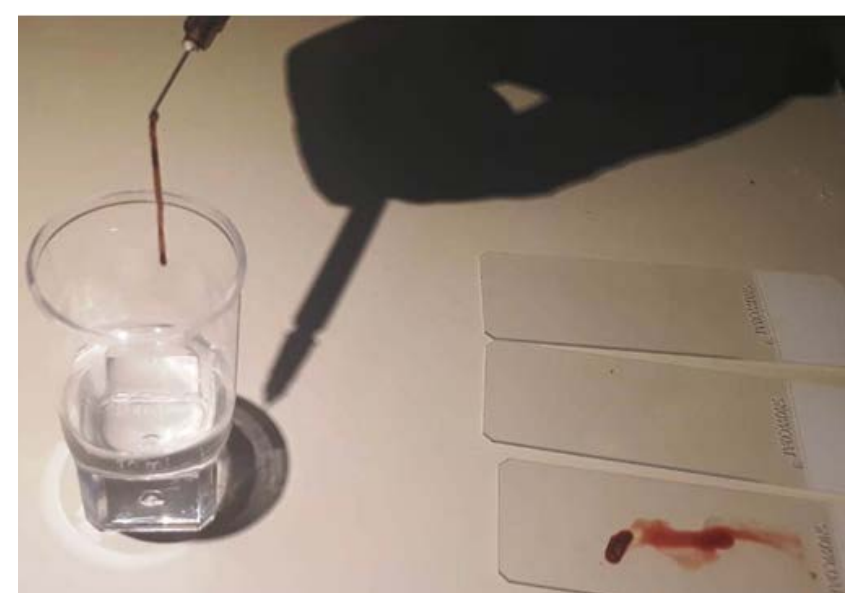

G

Fig. 2. An example of a thoracic ultrasonography (TUS) and shear wave elastography examination followed by ultrasonography-guided transthoracic needle biopsy.

A. Computed tomography scan of a left lung subpleural lesion that proved to be malignant (arrowhead) is shown. B. Ultrasonography with a convex probe $(6 \mathrm{MHz})$ of the same nodular lesion $(2.1 \mathrm{~cm})$ shows an irregular hypoechoic pattern. C. Color-coded two dimensional elastography map of the lesion (arrow) is created. D. Elastographic measurements were performed. On the monitor, in the left, are displayed 10 consecutive shear wave measurement in $\mathrm{m} / \mathrm{s}$, their median value and the interquartile range/median ratio (IQR/M). During each measurement a small fixed region of interest (ROI) of $1 \times 1 \mathrm{~cm}$ (arrow) was selected. The relative three dimensional elastography map is colorcoded based on the amount of tissue displacement in the selected ROI: tissue stiffness is indicated by a blue color occupying $>85 \%$ of the area. E. Convex probe with a central hole for needle insertion is shown. F. TUS-guided needle biopsy was performed. It is possible to see the needle tip in the nodule (arrow). G. Biopsy specimen to obtain sufficient material for both cytology and histology analyses is shown. 


\section{Results}

We enrolled 190 consecutive patients with peripheral pulmonary lesions (118 men, 72 women; aged $65.88 \pm 6.33$ years), of whom 129 showed multiple lesions on CT. In all the patients, TUS allowed identification of at least one area of subpleural consolidation, that was subjected to subsequent SWE examination $(n=190)$.

Of these patients, 124 (78 men, 46 women; aged 66.52 \pm 6.23 years) had primary lung carcinoma, 20 (15 men, 5 women; aged $66.65 \pm 4.58$ years) were diagnosed with lung metastasis, and 46 (25 men, 21 women; aged $63.72 \pm 7.19$ years) had pneumonia. The groups did not differ significantly in term of sex $(P=0.273)$ or smoking habit $(P=0.345)$. The patients with pneumonia were younger than those patients with primary lung carcinoma and lung metastasis $(P=0.034)$.

On CT scans, we found no significant differences in terms of air bronchogram and associated pleural effusion between benign and malignant lesions ( $P=0.881$ and $P=0.166$, respectively). However, necrotic areas were detected significantly more frequently in the pneumonia group $(P=0.001)$. The lesions presented no significant differences in terms of TUS characteristics suggestive of malignancy, such as irregular margins and inhomogeneous echogenicity $(P=0.598$ and $P=0.051$, respectively). The average diameter of subpleural lesions by TUS was $2.84 \pm 0.76 \mathrm{~cm}$ for primary lung carcinomas, $2.39 \pm 0.38 \mathrm{~cm}$ for metastases, and $2.88 \pm 0.51 \mathrm{~cm}$ for pneumonia; lung metastases were significantly smaller than primary lung carcinomas and pneumonia ( $P=0.016)$ (Table 1).

US-TNB was used to assess 135 lesions suspected of malignancy. Specimens of sufficient size and quality to reach a diagnosis were obtained in almost all patients. Macroscopically inadequate samples were obtained in four patients, requiring the repetition of the biopsy, which was immediately performed during the same session. Routine chest $X$-rays after US-TNB revealed only one case of partial pneumothorax, which spontaneously resolved.

The values of shear wave speed $(\mathrm{m} / \mathrm{s})$ and the Young modulus $(\mathrm{kPa})$ showed no statically significant differences between malignant

Table 1. Clinical characteristics of the patients in the study

\begin{tabular}{|c|c|c|c|c|}
\hline Variable & $\operatorname{PLC}(n=124)$ & $\operatorname{LM}(n=20)$ & PM $(n=46)$ & P-value \\
\hline Age (year) & $66.52 \pm 6.23$ & $66.65 \pm 4.58$ & $63.72 \pm 7.19$ & 0.034 \\
\hline \multicolumn{5}{|l|}{ Sex } \\
\hline Female & $46(37.1)$ & $5(25.0)$ & $21(45.7)$ & \\
\hline Smokers & $79(63.7)$ & $16(80.0)$ & $29(63.0)$ & 0.345 \\
\hline \multicolumn{5}{|l|}{ CT findings } \\
\hline Diameter (cm) & $3.33 \pm 0.88$ & $2.72 \pm 0.40$ & $3.26 \pm 0.57$ & 0.006 \\
\hline Pleural effusion & $61(49.2)$ & $14(70.0)$ & $27(58.7)$ & 0.166 \\
\hline Necrosis & $22(17.7)$ & $3(15.0)$ & $20(43.5)$ & 0.001 \\
\hline Air bronchogram & $22(17.7)$ & $4(20.0)$ & $7(15.2)$ & 0.881 \\
\hline Non-homogeneous pattern & $62(50.0)$ & $9(45.0)$ & $32(69.6)$ & 0.051 \\
\hline Irregular margins & $58(46.8)$ & $11(55.0)$ & $25(54.3)$ & 0.598 \\
\hline \multicolumn{5}{|l|}{ Position } \\
\hline Posterior-basal & $52(41.9)$ & $11(55.0)$ & $20(43.5)$ & 0.554 \\
\hline Posterior-medial & 19 (15.3) & $2(10.0)$ & $5(10.9)$ & 0.667 \\
\hline Lateral-basal & $31(25.0)$ & $1(5.0)$ & $13(28.3)$ & 0.106 \\
\hline Lateral-medial & $17(13.7)$ & $5(25.0)$ & $4(8.7)$ & 0.211 \\
\hline Anterior-medial & $4(3.2)$ & 0 & $2(4.3)$ & 0.652 \\
\hline
\end{tabular}

Values are presented as mean \pm SD or number (\%).

PLC, primary lung carcinoma; LM, lung metastasis; PM, pneumonia; CT, computed tomography; US, ultrasonography; SD, standard deviation. 
and benign lesions $(2.59 \pm 0.55 \mathrm{~m} / \mathrm{s}$ vs. $2.73 \pm 0.60 \mathrm{~m} / \mathrm{s}, P=0.143$ and $22.32 \pm 8.97 \mathrm{kPa}$ vs. $24.87 \pm 10.64 \mathrm{kPa}, \mathrm{P}=0.111$ ). We found no statistical difference in stiffness, both in terms of shear wave speed $(P=0.296)$ and the Young modulus $(P=0.280)$, between primary lung carcinomas, lung metastases, and pneumonia. Although pneumonia unexpectedly showed slightly higher stiffness values than primary lung carcinomas and lung metastases, the pairwise post-hoc Tukey test showed no significant difference between the mean of all possible pairs (Table 2).

Among the 124 subjects who received a histological diagnosis of primary lung carcinoma, the histological diagnoses were small cell lung carcinoma (7 cases), squamous cell carcinoma (27 cases); adenocarcinoma (55 cases) and large cell undifferentiated carcinoma (37 cases). In 20 patients, the final diagnosis was lung metastasis. There was no statistically significant difference in stiffness, either in terms of shear wave speed $(\mathrm{P}=0.393)$ or the Young modulus $(\mathrm{P}=0.536)$, between different histological types of primary lung carcinoma (Table 2).

Of the patients with pneumonia $(n=46), 22(48 \%)$ presented with classical features of CAP (i.e., fever, productive cough, malaise, and/ or dyspnea) and improved clinically within a few days of broadspectrum empirical antibiotic therapy, while 24 (52\%) presented persistent illnesses and consolidations despite an adequate antibiotic treatment duration of 10-14 days. In 13 of those patients, fiberoptic bronchoscopy with bronchoalveolar lavage and appropriate cultures revealed an infection of multi-drug resistant bacteria (10 cases), atypical mycobacteria (2 cases), and tuberculosis (1 case), and resolution of clinical symptoms and radiological changes was achieved after appropriately targeted antibiotic therapy. In the remaining 11 cases, bronchoscopy with microbiological evaluation was non-diagnostic and the patients underwent US-TNB, showing on histology the presence of a mixture of chronic inflammatory cells and fibroblasts within airspaces (organizing pneumonia). Secondline antibiotic therapy was prescribed, leading to resolution in all cases.

We found a statistically significant difference both in terms of shear wave speed and the Young modulus between usual CAP and $R-C A P$, with R-CAP showing higher stiffness $(2.43 \pm 0.40 \mathrm{~m} / \mathrm{s} \mathrm{vs}$. $3.03 \pm 0.63 \mathrm{~m} / \mathrm{s}, P<0.001$ and $19.40 \pm 6.45 \mathrm{kPa}$ vs. $30.45 \pm 11.78$ $\mathrm{kPa}, \mathrm{P}<0.001$ ) (Table 2).

Comparing subpleural lesions with and without necrosis on $\mathrm{CT}$, we found slightly lower stiffness in lesions with necrosis. Although this difference was not statistically relevant for primary lung carcinomas and metastases, the presence of necrosis significantly reduced both the values of shear wave speed $(P=0.002)$ and the Young modulus $(\mathrm{P}<0.001)$ in the pneumonia group (Table 3$)$. Restricting the analysis to lesions without necrosis on $\mathrm{CT}$, pneumonia showed higher
Table 2. Comparison of shear wave speed $(\mathrm{m} / \mathrm{s})$ and the Young modulus $(\mathrm{kPa})$ between PLC, LM, and PM, between different histological types of PLC, and between usual and R-CAP

\begin{tabular}{|c|c|c|}
\hline & Shear wave speed (m/s) & Young modulus (kPa) \\
\hline \multicolumn{3}{|l|}{ Total } \\
\hline $\operatorname{PLC}(n=124)$ & $2.58 \pm 0.55(1.20-4.00)$ & $22.31 \pm 9.05(4.40-50.30)$ \\
\hline $\operatorname{LM}(\mathrm{n}=20)$ & $2.60 \pm 0.50(1.50-3.60)$ & $22.39 \pm 8.44(7.10-40.70)$ \\
\hline$P M(n=46)$ & $2.73 \pm 0.60(1.10-4.20)$ & $24.87 \pm 10.64(3.80-55.40)$ \\
\hline P-value & 0.296 & 0.280 \\
\hline \multicolumn{3}{|l|}{$\begin{array}{l}\text { Primary lung } \\
\text { carcinoma }\end{array}$} \\
\hline $\operatorname{SCLC}(n=7)$ & $2.63 \pm 0.57(1.70-3.80)$ & $23.09 \pm 9.34(9.10-45.40)$ \\
\hline $\operatorname{SCC}(n=25)$ & $2.42 \pm 0.59(1.20-4.00)$ & $20.02 \pm 9.48(4.50-50.30)$ \\
\hline $\operatorname{ADC}(n=55)$ & $2.64 \pm 0.53(1.50-3.80)$ & $23.13 \pm 8.84(7.10-45.40)$ \\
\hline UDLC $(n=37)$ & $2.61 \pm 0.52(1.60-3.80)$ & $22.61 \pm 8.67(8.00-45.40)$ \\
\hline P-value & 0.393 & 0.536 \\
\hline \multicolumn{3}{|l|}{ Pneumonia } \\
\hline CAP $(n=22)$ & $2.43 \pm 0.40(1.10-3.30)$ & $19.40 \pm 6.45(3.80-34.20)$ \\
\hline$R-C A P(n=24)$ & $3.03 \pm 0.63(1.90-4.20)$ & $30.45 \pm 11.78(11.30-55.40)$ \\
\hline P-value & $<0.001$ & $<0.001$ \\
\hline
\end{tabular}

Values are presented as mean \pm SD (min-max).

PLC, primary lung carcinoma; LM, lung metastasis; PM, pneumonia; R-CAP, refractory to standard treatment community-acquired pneumonia; SCLC, small cell lung cancer; SCC, squamous cell lung carcinoma; ADC, adenocarcinoma; UDLC, undifferentiated lung carcinoma; CAP, community-acquired pneumonia; SD, standard deviation.

stiffness than primary lung cancers $(2.95 \pm 0.68 \mathrm{~m} / \mathrm{s}$ vs. $2.60 \pm 0.54$ $\mathrm{m} / \mathrm{s}, \mathrm{P}=0.006 ; 29.24 \pm 12.28 \mathrm{kPa}$ vs. $22.46 \pm 8.75 \mathrm{kPa}, \mathrm{P}=0.002$ ).

In addition, we found a statistically significant difference in terms of shear wave speed and the Young modulus between lesions without and with CT pleural effusion in primary lung carcinomas $(P=0.004)$ and in metastases $(P=0.002)$. In the pneumonia group, we recorded a slightly lower stiffness in lesions with pleural effusion, but this difference was not statistically significant $(P=0.259)$ (Table 3). Data from the SWE assessment of the 25 healthy volunteers are reported in Table 4.

\section{Discussion}

Few studies have investigated the application of UE in pulmonary lesions and pulmonary diseases, and UE does not yet have any clinical indications for the lung.

Compared to SE, point SWE has the advantage of not generating shear by applying a mechanical vibration to the surface of the body, but by focusing an acoustic radiation force (or acoustic "push pulse") inside the tissue of interest, thereby reducing the subjective influence of the operator. This acoustic push pulse moves tissue back and forth in a direction parallel to the incident ultrasonic 
Table 3. Sub-analysis of shear wave speed $(\mathrm{m} / \mathrm{s})$ and the Young modulus $(\mathrm{kPa})$ in lesions with and without necrosis and with and without pleural effusion on CT among PLCs, LMs, and PM

\begin{tabular}{|c|c|c|c|c|c|c|c|c|c|}
\hline \multirow{2}{*}{ Subgroup } & \multicolumn{3}{|c|}{ PLC } & \multicolumn{3}{|c|}{ LM } & \multicolumn{3}{|c|}{ PM } \\
\hline & No & Yes & P-value & No & Yes & P-value & No & Yes & P-value \\
\hline \multicolumn{10}{|l|}{ Necrosis } \\
\hline No. & 102 & 22 & & 17 & 3 & & 26 & 20 & \\
\hline Shear wave speed (m/s) & $2.60 \pm 0.54$ & $2.42 \pm 0.50$ & 0.154 & $2.58 \pm 0.52$ & $2.36 \pm 0.15$ & 0.485 & $2.95 \pm 0.68$ & $2.43 \pm 0.34$ & 0.002 \\
\hline Young modulus (kPa) & $22.46 \pm 8.75$ & $19.68 \pm 8.37$ & 0.176 & $22.19 \pm 8.71$ & $17.68 \pm 2.37$ & 0.394 & $29.24 \pm 12.28$ & $18.97 \pm 5.52$ & $<0.001$ \\
\hline \multicolumn{10}{|l|}{ Pleural effusion } \\
\hline No. & 63 & 61 & & 6 & 14 & & 19 & 27 & \\
\hline Shear wave speed (m/s) & $2.75 \pm 0.47$ & $2.47 \pm 0.59$ & 0.004 & $3.20 \pm 0.40$ & $2.45 \pm 0.45$ & 0.002 & $2.91 \pm 0.63$ & $2.67 \pm 0.64$ & 0.214 \\
\hline Young modulus (kPa) & $24.71 \pm 7.84$ & $20.71 \pm 9.38$ & 0.011 & $32.73 \pm 7.98$ & $19.81 \pm 7.03$ & 0.002 & $28.06 \pm 11.99$ & $24.14 \pm 11.05$ & 0.259 \\
\hline
\end{tabular}

$\mathrm{CT}$, computed tomography; PLC, primary lung carcinoma; LM, lung metastasis; PM, pneumonia.

Table 4. Clinical characteristics and SWE values in 25 healthy volunteers

\begin{tabular}{lc}
\hline \multicolumn{1}{c}{ Healthy volunteer } & Value \\
\hline No. & 25 \\
Age (year) & $66.52 \pm 6.23$ \\
Sex & \\
Male & $13(52.0)$ \\
Female & $12(48.0)$ \\
Smokers & 0 \\
Shear wave speed $(\mathrm{m} / \mathrm{s})$ & $14.64 \pm 2.19$ \\
Young modulus $(\mathrm{kPa})$ & $692.53 \pm 202.84$ \\
\hline
\end{tabular}

Values are presented as number (\%) or mean \pm SD.

SWE values were calculated as the average between two medial-anterior hemiclavicular and posterior median hemiscapular scans.

SWE, shear wave elastography; SD, standard deviation.

compression wave, generating within the lesion a shear wave that propagates in a perpendicular direction [22]. A quantitative tissue stiffness measurement can be obtained by tracking the shear wave speed (cs) in the examined lesion with the single-element ultrasound transducer and converting it into the Young modulus using the relationship $\mathrm{E}=3 \rho \mathrm{c}_{\mathrm{s}}^{2}$. In this study, we have chosen to report both shear wave speed (in $\mathrm{m} / \mathrm{s}$ ) and the Young modulus (in $\mathrm{kPa}$ ) in order to facilitate comparison with the different data presented in the literature.

In a recent study, Ozgokce et al. [23] reported that TUS-SWE had a sensitivity and a specificity of $97.7 \%$ in differentiating between benign and malignant subpleural lesions when a speed of $2.47 \mathrm{~m} / \mathrm{s}$ was selected as the cut-off value, while Wei et al. [24] computed a sensitivity of $70.9 \%$ and a specificity of $69.4 \%$ when the cut-off value was set at $1.951 \mathrm{~m} / \mathrm{s}$. In our study, the mean value of shear wave speed in primary lung carcinomas was $2.58 \pm 0.55 \mathrm{~m} / \mathrm{s}$, with a modal value of $2.90 \mathrm{~m} / \mathrm{s}$, but we recorded no statistically significant differences either in the shear wave speed (in $\mathrm{m} / \mathrm{s}$ ) or the Young modulus (in $\mathrm{kPa}$ ) between primary lung carcinomas, lung metastases, and pneumonia. Kuo et al. [25] reported that in TUS-SWE evaluation, among pulmonary malignancies, the elasticity of pulmonary adenocarcinomas (in $\mathrm{kPa}$ ) was significantly higher than that of the non-adenocarcinoma malignancies $(87.90 \pm 26.35$ vs. $51.80 \pm 17.40$, $P<0.01)$. On the contrary, in our study, statistically significant differences in terms of shear wave speed (in $\mathrm{m} / \mathrm{s}$ ) and the Young modulus (in $\mathrm{kPa}$ ) were not recorded between different histological types of primary lung carcinoma (Fig. 3).

Despite having negative results, the principal strength of our study is that it shed light on the current technical limitations that hinder the reproducibility of TUS-SWE measurements in lung lesions. Point SWE relies on the assumption that the examined tissue is elastic (i.e., that after stress cessation, the tissue returns to its original nondeformed equilibrium state, independently from the applied stress rate), incompressible (i.e., the overall volume of the tissue remains the same under applied stress) and isotropic (i.e., the tissue is symmetrical/homogeneous and responds to stress in the same way from all directions) [26]. However, this ideal physical model cannot be perfectly applied to the reality of soft tissues.

First, the assumption of elasticity is frequently violated, especially in tumors, which have a highly heterogeneous microenvironment containing regions with both an elastic (i.e., fibrosis and calcifications) and a viscous (i.e., blood pools, cystic degeneration areas, and necrosis) mechanical response when probed. Interestingly, Wei et al. [24] reported that tuberculosis (four cases) and chronic inflammation (one case) had a shear wave speed on acoustic radiation force imaging comparable to that of malignant lesions. This phenomenon was explained by authors in terms of the formation of lung fibrous band structures in tuberculosis and the proliferation 


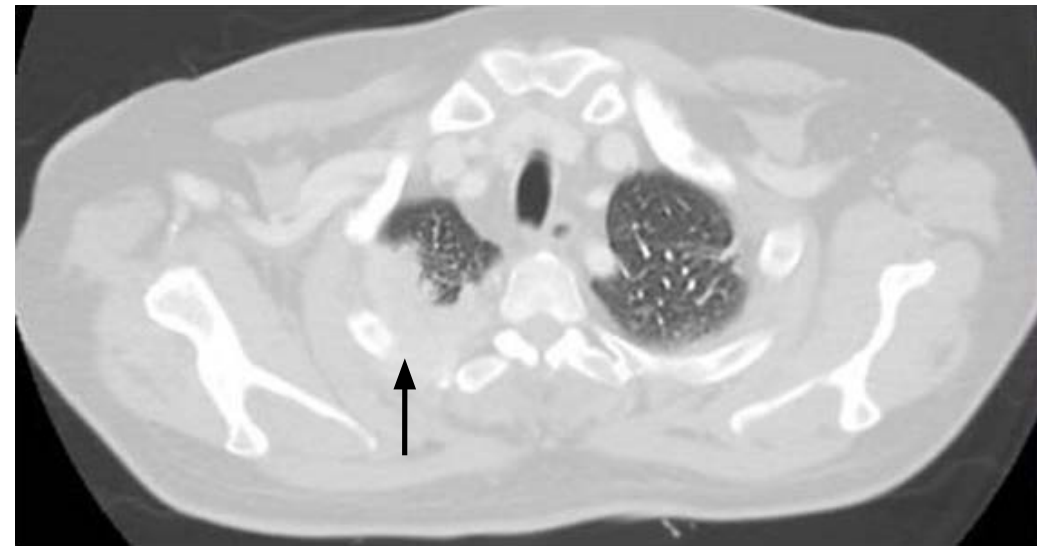

A

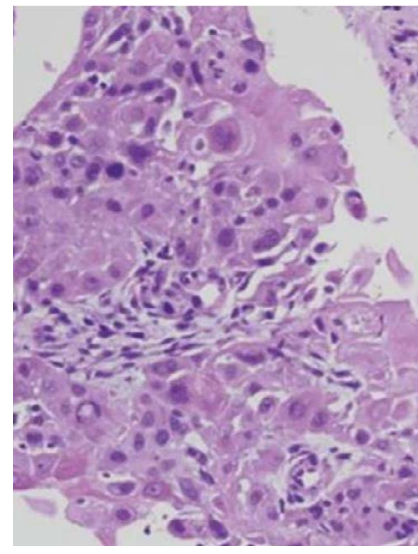

B

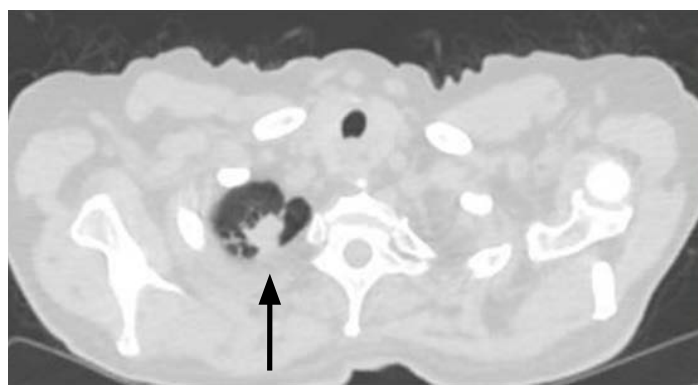

D

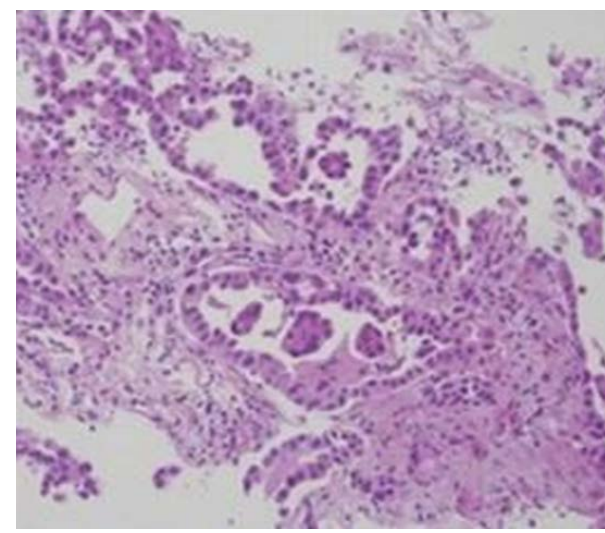

E

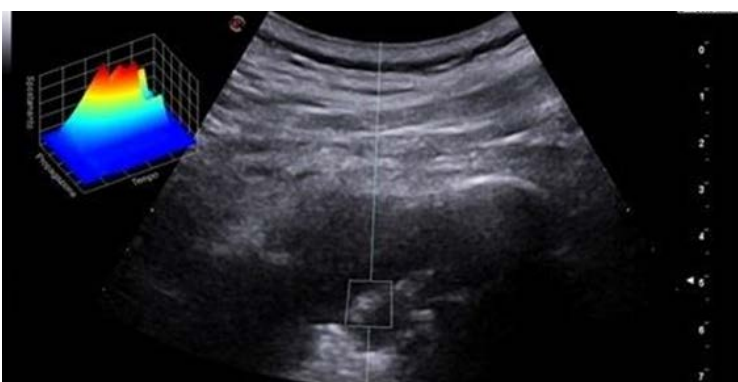

C

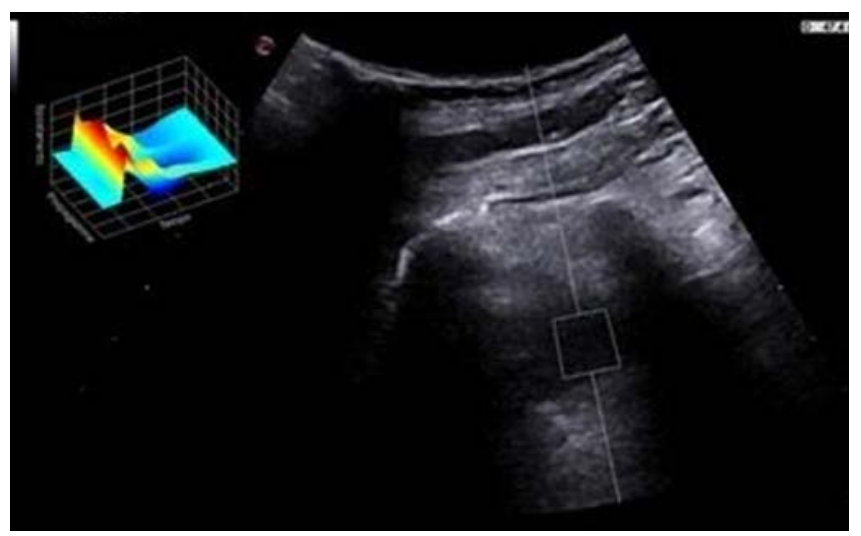

$\mathrm{F}$

Fig. 3. Shear wave elastography (SWE) examination of malignant lesions.

A. Computed tomography (CT) scan shows a lung lesion suspected of malignancy in the posterior upper right lobe (arrow). B. Histology reveals a diagnosis of lung squamous cell carcinoma (H\&E, $\times 600$ ). C. B-mode ultrasound (US) using a convex probe (6 MHz) shows an irregular subpleural consolidation with an inhomogeneous pattern. The three-dimensional elastography map in the left side is color-coded based on the amount of tissue displacement: the center of the region of interest shows high strain, encoded as red (soft tissue), while its peripheral part is blue (low strain, stiff tissue). The mean SWE value in the entire lesion was $2.8 \mathrm{~m} / \mathrm{s}$. D. CT scan shows a lung subpleural lesion in the apical upper right lobe (arrow). E. Histology reveals a diagnosis of lung adenocarcinoma (H\&E, $\times 600)$. F. US imaging with a convex probe $(6 \mathrm{MHz})$ shows an irregular mixed hypoechoic and hyperechoic pattern. The relative three-dimensional color-coded US elastography map of the region of interest shows an asymmetrical area of soft tissue (red) on a light blue background (intermediate stiff tissue). The mean SWE value in the entire lesion was $2.6 \mathrm{~m} / \mathrm{s}$. 
of fibroblast foci in chronic inflammation, which might increase the tissue stiffness. Additionally, one small cell lung carcinoma showed a mean shear wave speed in the range of benignity $(1.20 \mathrm{~m} / \mathrm{s})$, and this result was explained by appealing to the complex heterogeneity of tumor cells. In our case series, pneumonia seemed to show a greater stiffness than primary lung carcinomas. This probably occurred because we included most cases of chronic pneumonia, in which the presence of fibrotic organization may have played an important role in influencing stiffness assessments. As confirmation, we recorded a significantly higher stiffness in R-CAP, and the biopsy examination of 11 persistent lesions revealed on histology a mixed interstitial infiltrate of chronic inflammatory cells and fibroblast proliferation (Fig. 4).

Restricting the analysis to subpleural lesions without necrosis on $\mathrm{CT}$, the difference in stiffness between primary lung carcinomas and pneumonia became statistically significant, with pneumonia showing greater stiffness. Indeed, necrotic areas were significantly more frequently detected in the pneumonia group. These data may confirm that fibrosis and necrosis are a source of bias in UE examinations. More specifically, the presence of fibrotic foci may

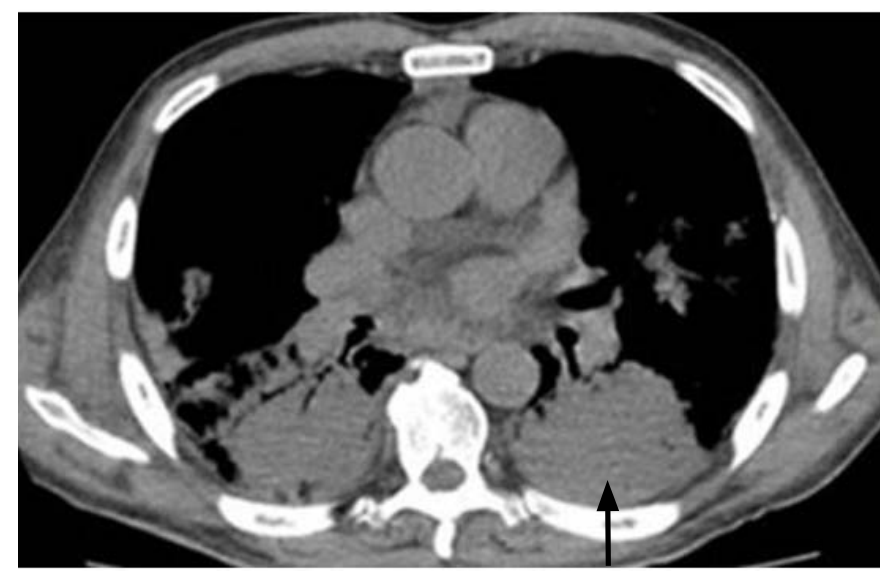

A

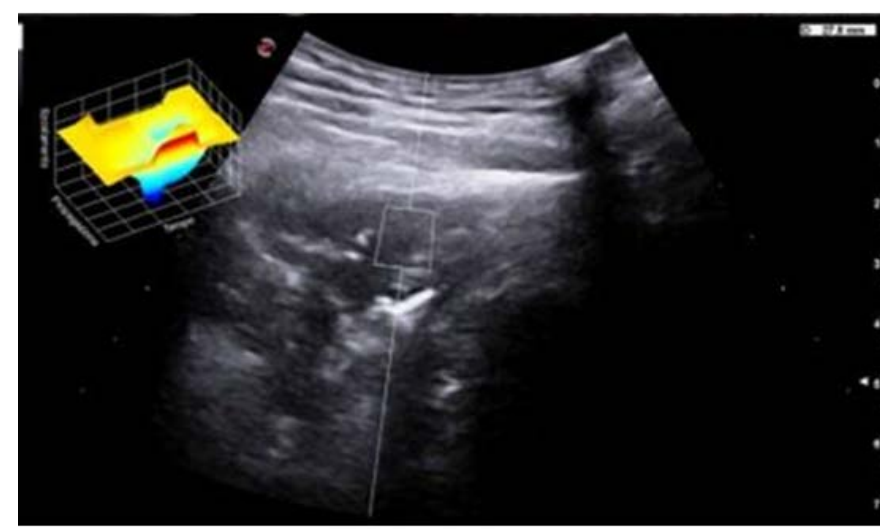

C have influenced the results by increasing tissue stiffness; however, necrosis might have reduced stiffness (Fig. 5).

Second, incompressibility is violated when tissues lose volume when compressed (i.e., fluids) and shear waves cannot travel through fluids because fluids do not support shear [27]. As a result, the interposition of effusion between the tissue and the tracking probe may decrease the shear wave signal as a function of depth, thereby limiting the accurate assessment of deeper tissues or organs. It is for this reason that ascites is indicated as a physical limitation in SWE measurements of cirrhotic liver stiffness [28]. Interestingly, in our study we found a statistically significant reduction in the shear wave velocity and Young modulus in lesions with pleural effusion compared to those without pleural effusion, both in primary lung carcinomas and lung metastases. Therefore, the presence of pleural effusions or, in general, of fluid/semifluid content in necrotic areas may invalidate the elastographic results. Furthermore, no parenchymatous tissue is perfectly incompressible. As a result, even in SWE mode, excessive pressure applied by the transducer on the chest during data acquisition may cause crushing of the underlying tissues, resulting in a precompression artefact, which consists of a

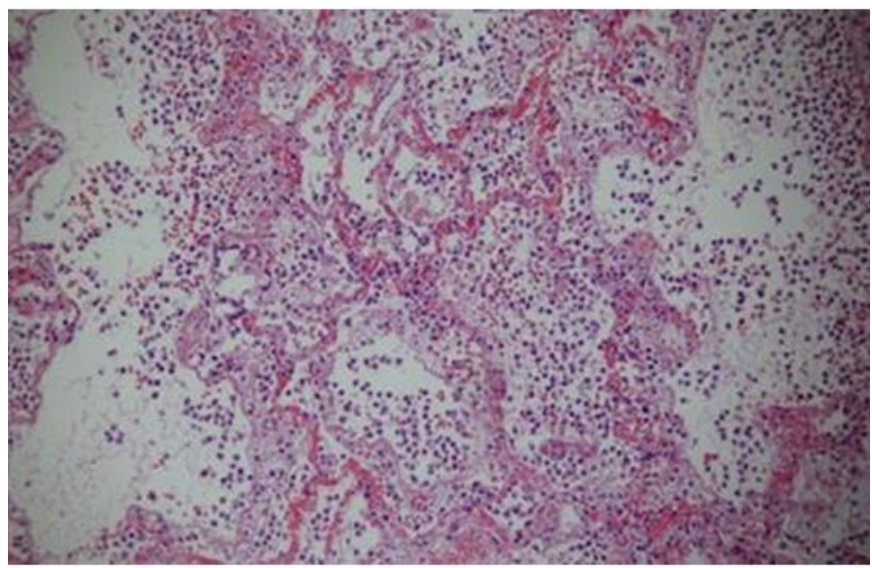

B

Fig. 4. Shear wave elastography (SWE) examination in pneumonia.

A. Computed tomography scan shows a bilateral pneumonia. The left lesion (arrow) was selected for subsequent examinations. B. Histology reveals a diagnosis of chronic pneumonia showing a mixture of chronic inflammatory cells and fibroblasts within airspaces (H\&E, $\times 200)$. C. The B-mode ultrasound (US) using a convex probe $(6 \mathrm{MHz})$ shows hypoechoic/anechoic consolidation with hyperechoic spots/striae within. The relative three-dimensional US elastography color-coded map of the region of interest shows a central area of low strain (hard tissue), encoded as blue, surrounded by a peripheral area of intermediate stiffness (yellow). The mean SWE value of the entire lesion was $3.3 \mathrm{~m} / \mathrm{s}$. 


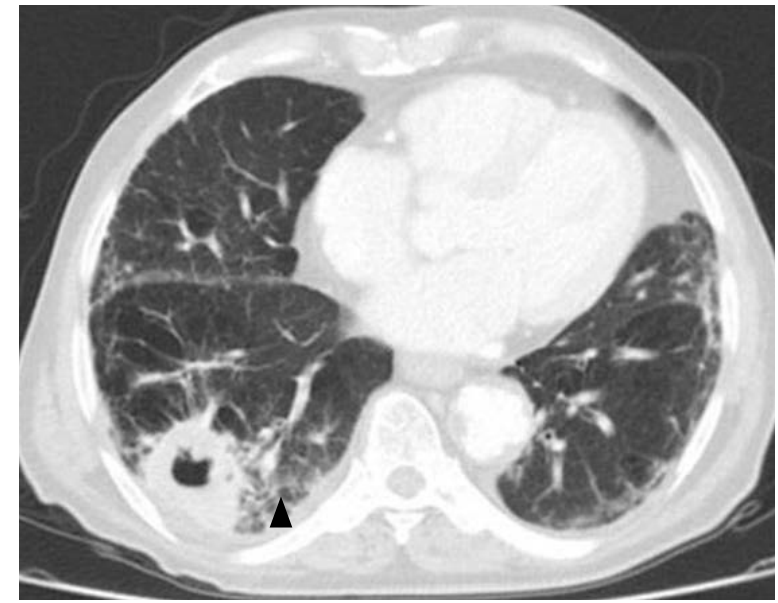

A

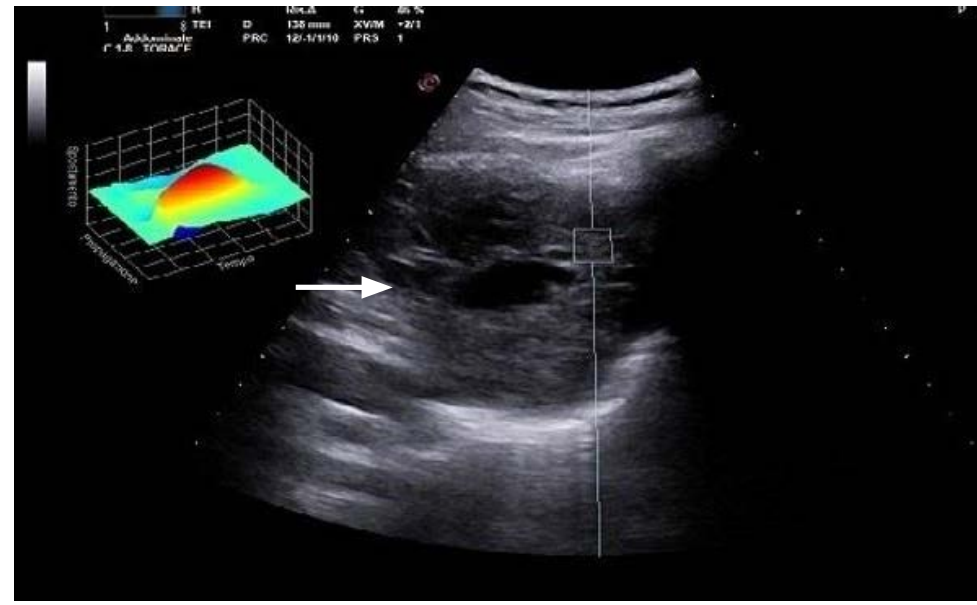

B

Fig. 5. Shear wave elastography (SWE) examinations in a necrotic lesion.

A. Computed tomography scan shows a lung lesion with central necrosis in the lower right lobe (arrowhead). B. The B-mode ultrasound (US) using a convex probe (6 MHz) shows a hypoechoic consolidation with hyperechoic spots/striae within and a central anechoic area (arrow). The relative three-dimensional US elastography color-coded map of the region of interest shows a central area of high strain encoded as red (soft tissue) on a background of intermediate stiffness encoded as green/light blue. The mean SWE value in the entire lesion was $2.7 \mathrm{~m} / \mathrm{s}$.

false finding of uniformly increased shear wave speed associated with decreased elastogram contrast between areas of different tissue stiffness [29].

Third, the assumption that tissue is isotropic (homogeneous) is violated at tissue interfaces. For example, the interposition of even a micron of normal aerated lung parenchyma between the lesion and the pleural surface or within the lesion (i.e., air bronchogram) may lead to incorrect speed estimates. In human soft tissues, ultrasonic compression waves propagate at around $1,500 \mathrm{~m} / \mathrm{s}[22]$, but in the air medium, their velocity is dramatically lowered to 340 $\mathrm{m} / \mathrm{s}$. As a result, near solid/air interfaces the incident ultrasonic compression wave is reflected according to Snell's law and some of the compression energy is converted into shear energy. The very high values obtained both in terms of shear wave speed $(\mathrm{m} / \mathrm{s})$ and the Young modulus $(\mathrm{kPa})$ in our 25 healthy volunteers were probably due to compression wave reflection at the interface between the chest wall and the lung air content. This suggests, as a further limitation, that TUS-SWE cannot assess the presence of even a very large space-occupying lesion when even a very thin layer of air blocks the signal (exactly as in conventional TUS B-mode examinations) [1]. Similarly, increased stiffness may be found in chronic obstructive pulmonary disease (COPD) patients at the interface with areas of smoke-induced remodeling in peripheral airways, while traction cysts/honeycombing areas may decay wave propagation, resulting in SWE artifacts.

Fourth, if a patient fails to hold his or her breath during the exam, this may result in a sliding artifact, which consists in an erroneous shear wave speed estimation due to the lesion movement in and out of the imaging plane while the elastogram is being obtained [29].

Last but not least, another important limitation of the assessment of pulmonary lesions by TUS-SWE lies in the fact that its protocol is standardized for the liver [21]. Use for other organs is still offlabel and not validated, and protocols in the lung vary among the currently available studies in the literature.

In conclusion, our experience seems to demonstrate that TUS-SWE is not able to reliably distinguish lung malignancies from pneumonia or between different histological types of lung carcinoma. In particular, TUS-SWE cannot discern the nature of chronic subpleural consolidations, which represent an ideal target for a noninvasive assessment due to the clinical dilemma of their management. The clinical usefulness of lung SWE seems to be currently limited by the concomitant presence of pleural effusion, fibrosis, calcification, necrosis, fat, or air. It is likely that TUS-SWE may represent a good complementary noninvasive imaging modality for assessing increased stiffness in diffuse interstitial lung disease characterized by an early involvement of the subpleural interstitium. However, further studies with a larger scale and using more standardized methodologies are still needed to assess the potential role of TUSSWE.

ORCID: Carla Maria Irene Quarato: https://orcid.org/0000-0003-4299-7403; Mariapia Venuti: https://orcid.org/0000-0002-6579-4601; Donato Lacedonia: https://orcid.org/00000003-0429-7967; Antonio Mirijello: https://orcid.org/0000-0003-3932-3803; Salvatore De Cosmo: https://orcid.org/0000-0001-8787-8286; Giulia Scioscia: https://orcid.org/00000002-2667-077X; Maria Pia Foschino Barbaro: https://orcid.org/0000-0001-9939-2697; 
Massimiliano Copetti: https://orcid.org/0000-0002-7960-5947; Marco Sperandeo: https://orcid.org/0000-0001-6685-6942

\section{Author Contributions}

Conceptualization: Quarato CMI, Venuti M, Sperandeo M. Data acquisition: Quarato CMI, Venuti M, Dimitri L, Lacedonia D, Simeone A, Mirijello A, Maiello E, Taurchini M, Sperandeo M. Data analysis or interpretation: Quarato $\mathrm{CMI}$, Venuti $\mathrm{M}$, Dimitri L, Lacedonia D, Simeone A, Mirijello A, De Cosmo S, Maiello E, Taurchini M, Scioscia G, Barbaro MPF, Copetti M, Sperandeo M. Drafting of the manuscript: Quarato CMI, Sperandeo M. Critical revision of the manuscript: Quarato CMI, Venuti M, Dimitri L, Lacedonia D, Simeone A, Mirijello A, De Cosmo S, Maiello E, Taurchini M, Scioscia G, Barbaro MPF, Copetti M, Sperandeo M. Approval of the final version of the manuscript: all authors.

\section{Conflict of Interest}

No potential conflict of interest relevant to this article was reported.

\section{References}

1. Sperandeo M, Rotondo A, Guglielmi G, Catalano D, Feragalli $B$, Trovato GM. Transthoracic ultrasound in the assessment of pleural and pulmonary diseases: use and limitations. Radiol Med 2014;119:729-740.

2. Rodriguez EF, Pastorello R, Osmani L, Hopkins M, Kryatova M, Kawamoto $S$, et al. Ultrasound-guided transthoracic fine-needle aspiration: a reliable tool in diagnosis and molecular profiling of lung masses. Acta Cytol 2020;64:208-215.

3. Sperandeo M, Dimitri L, Pirri C, Trovato FM, Catalano D, Trovato GM. Advantages of thoracic ultrasound-guided fine-needle aspiration biopsy in lung cancer and mesothelioma. Chest 2014;146:e178-e179.

4. Kirtland SH, Winterbauer RH. Slowly resolving, chronic, and recurrent pneumonia. Clin Chest Med 1991;12:303-318.

5. Alves dos Santos JW, Torres A, Michel GT, de Figueiredo CW, Mileto JN, Foletto VG Jr, et al. Non-infectious and unusual infectious mimics of community-acquired pneumonia. Respir Med 2004;98:488-494.

6. Sperandeo M, Trovato FM, Dimitri L, Catalano D, Simeone A, Martines $G F$, et al. Lung transthoracic ultrasound elastography imaging and guided biopsies of subpleural cancer: a preliminary report. Acta Radiol 2015;56:798-805.

7. Ferraioli $G$, Parekh P, Levitov AB, Filice C. Shear wave elastography for evaluation of liver fibrosis. J Ultrasound Med 2014;33:197-203.

8. Samir AE, Dhyani M, Vij A, Bhan AK, Halpern EF, Mendez-Navarro $J$, et al. Shear-wave elastography for the estimation of liver fibrosis in chronic liver disease: determining accuracy and ideal site for measurement. Radiology 2015;274:888-896.

9. Chang JM, Park IA, Lee SH, Kim WH, Bae MS, Koo HR, et al. Stiffness of tumours measured by shear-wave elastography correlated with subtypes of breast cancer. Eur Radiol 2013;23:2450-2458.

10. Lee SH, Chang JM, Kim WH, Bae MS, Cho N, Yi A, et al. Differentiation of benign from malignant solid breast masses: comparison of two-dimensional and three-dimensional shear-wave elastography. Eur Radiol 2013;23:1015-1026.

11. Boehm K, Salomon G, Beyer B, Schiffmann J, Simonis K, Graefen $M$, et al. Shear wave elastography for localization of prostate cancer lesions and assessment of elasticity thresholds: implications for targeted biopsies and active surveillance protocols. J Urol 2015;193:794-800.

12. Liu B, Liang J, Zheng Y, Xie X, Huang G, Zhou L, et al. Twodimensional shear wave elastography as promising diagnostic too for predicting malignant thyroid nodules: a prospective singlecentre experience. Eur Radiol 2015;25:624-634.

13. Liu BX, Xie XY, Liang JY, Zheng YL, Huang GL, Zhou LY, et al. Shear wave elastography versus real-time elastography on evaluation thyroid nodules: a preliminary study. Eur J Radiol 2014;83:11351143.

14. Bhatia KS, Cho CC, Tong CS, Yuen EH, Ahuja AT. Shear wave elasticity imaging of cervical lymph nodes. Ultrasound Med Bio 2012;38:195-201.

15. Chen XM, Cui LG, He P, Shen WW, Qian YJ, Wang JR. Shear wave elastographic characterization of normal and torn achilles tendons: a pilot study. I Ultrasound Med 2013;32:449-455.

16. Hatta T, Giambini H, Uehara K, Okamoto S, Chen S, Sperling JW, et al. Quantitative assessment of rotator cuff muscle elasticity: Reliability and feasibility of shear wave elastography. J Biomech 2015;48:3853-3858.

17. Franchi-Abella S, Elie C, Correas JM. Ultrasound elastography: advantages, limitations and artefacts of the different techniques from a study on a phantom. Diagn Interv Imaging 2013;94:497501.

18. Janczewska E, Pisula A, Simon K. Recommendations for elastography-based imaging of liver. Przegl Epidemiol 2015;69:317321, 429-333.

19. Postmus PE, Kerr KM, Oudkerk M, Senan S, Waller DA, Vansteenkiste J, et al. Early and locally advanced non-smallcell lung cancer (NSCLC): ESMO Clinical Practice Guidelines for diagnosis, treatment and follow-up. Ann Oncol 2017;28:iv1-iv21.

20. Ettinger DS, Akerley W, Borghaei H, Chang AC, Cheney RT, Chirieac LR, et al. Non-small cell lung cancer. J Natl Compr Canc Netw 2012;10:1236-1271.

21. Dietrich CF, Bamber J, Berzigotti A, Bota S, Cantisani V, Castera L, et al. EFSUMB guidelines and recommendations on the clinical use of liver ultrasound elastography, update 2017 (long version). Ultraschall Med 2017;38:e16-e47. 
22. Tang A, Cloutier G, Szeverenyi NM, Sirlin CB. Ultrasound elastography and MR elastography for assessing liver fibrosis: Part 1, principles and techniques. AJR Am J Roentgenol 2015;205:22-32.

23. Ozgokce M, Yavuz A, Akbudak I, Durmaz F, Uney I, Aydin Y, et al. Usability of transthoracic shear wave elastography in differentiation of subpleural solid masses. Ultrasound Q 2018;34:233-237.

24. Wei H, Lu Y, Ji Q, Zhou H, Zhou X. The application of conventional us and transthoracic ultrasound elastography in evaluating peripheral pulmonary lesions. Exp Ther Med 2018;16:1203-1208.

25. Kuo YW, Chen YL, Wu HD, Chien YC, Huang CK, Wang HC. Application of transthoracic shear-wave ultrasound elastography in lung lesions. Eur Respir J 2021;57:2002347.
26. Sigrist RM, Liau J, Kaffas AE, Chammas MC, Willmann JK. Ultrasound elastography: review of techniques and clinical applications. Theranostics 2017;7:1303-1329.

27. Szabo TL. Diagnostic ultrasound imaging: inside out. Amsterdam: Elsevier, 2014;55-80.

28. Mancini M, Salomone Megna A, Ragucci M, De Luca M, Marino Marsilia G, Nardone G, et al. Reproducibility of shear wave elastography (SWE) in patients with chronic liver disease. PLoS One 2017;12:e0185391.

29. Prabhu SJ, Kanal K, Bhargava P, Vaidya S, Dighe MK. Ultrasound artifacts: classification, applied physics with illustrations, and imaging appearances. Ultrasound Q 2014;30:145-157. 